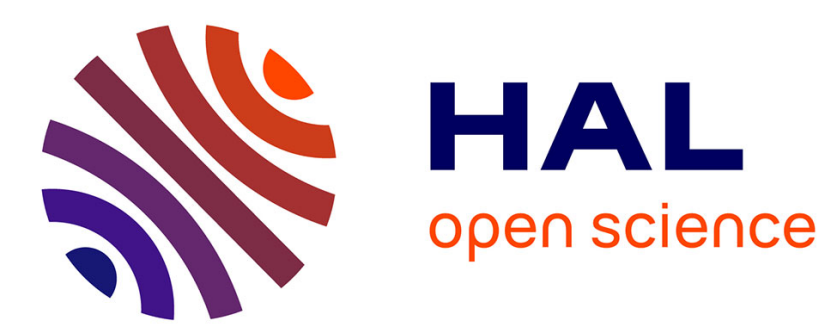

\title{
L'atelier de la Sarra
}

Colette Laroche

\section{To cite this version:}

Colette Laroche. L'atelier de la Sarra. Gallia - Archéologie de la France antique, 1997, Les productions des ateliers de potiers antiques de Lyon (2e partie) : les ateliers du Ier s. après J.-C., 54, pp.55-61. 10.3406/galia.1997.3236 . hal-01911499

\section{HAL Id: hal-01911499 \\ https://hal.science/hal-01911499}

Submitted on 29 Jan 2020

HAL is a multi-disciplinary open access archive for the deposit and dissemination of scientific research documents, whether they are published or not. The documents may come from teaching and research institutions in France or abroad, or from public or private research centers.
L'archive ouverte pluridisciplinaire HAL, est destinée au dépôt et à la diffusion de documents scientifiques de niveau recherche, publiés ou non, émanant des établissements d'enseignement et de recherche français ou étrangers, des laboratoires publics ou privés.

\section{(이) $\$$}

Distributed under a Creative Commons Attribution - NonCommercial - NoDerivatives| 4.0 


\title{
L'ATELIER DE LA SARRA
}

\author{
Colette LAROCHE
}

Résumé. En 1969, des trav'aux ont énentré plusieurs fours sur le plateau de la Sarra, ne Pauline-Jaricot. L'abondant matériel recueilli alteste une production de céramique culinnire cuite en mode $B$, datée de la première moitié du Irr $\mathrm{s}$.

Abstract. In 1969, a building site cut sereral kilns on the plateau de la Sarra, in Pauline-faricot street. A lot of pottery found there showed that this zookshop produced gren cooking zuares, dating from the first half of the l's century.

\section{EIRCONSTANCES DES DÉCOUVERTES}

(II)

L'atelier de la Sarra à Lyon a été découvert en 1\%69 par J. I asfargues (1973) lors de travaux urbains.

(1)

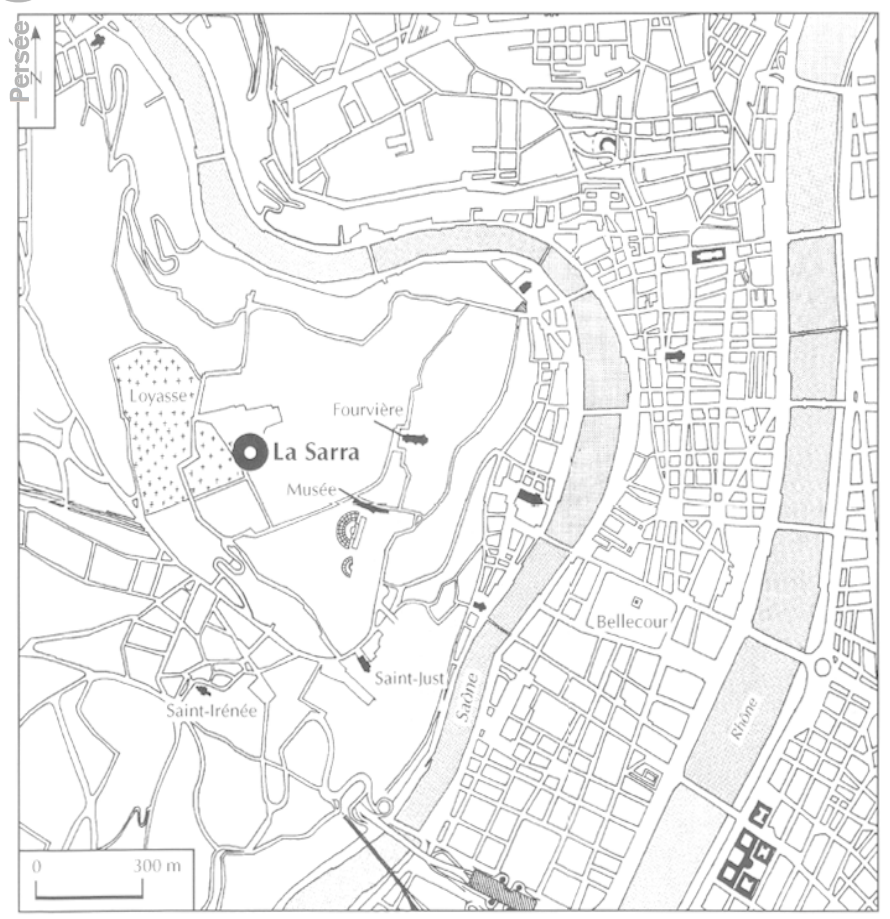

Fig. 20. Plan de localisation de l'atelier de la Sarra.
Il est situé sur le rebord nord du plateau de la Sarra de la colline de Fourvière (fig. 20). Deux fours primitifs, simples fosses rubćfiées et une lentille riche en céramiques, ont pu être fouillés. Outre la production de l'atelier, essenticllement de la céramique commune : 1178 tessons pour 112 vases (tabl. IV), le mobilier céramique comprend deux formes de sigillée (pl. 14, n" 1 et 2) ct une forme d'imitation de sigillée (Ritt. 5) (pl. 14, n"3).

Tabl. IV. Données quantitatives des vases de l'atelier de la Sarra.

\begin{tabular}{|l|r|r|r|}
\hline \multicolumn{1}{|c|}{ La Sarra } & fragments & vases & $\%$ vases \\
\hline Plats & 244 & 34 & 30 \\
\hline Marmites & 31 & 6 & 5 \\
\hline Brûle-parfum & 92 & 5 & 20 \\
\hline Couvercles & 74 & 22 & 15 \\
\hline Pots ovoïdes & 112 & 17 & 2 \\
\hline Pots cylindriques & 20 & 2 & 11 \\
\hline Pots carénés & 133 & 12 & 4 \\
\hline Pichets & 12 & 5 & 1 \\
\hline Cruches à bec tréflé & 57 & 4 & 3 \\
\hline Pot indéterminé & 1 & 1 & 1 \\
\hline Creusets & 3 & 3 & - \\
\hline Pot de fleurs & 1 & 1 & 0 \\
\hline Fragments non attribués & 398 & 0 & 112 \\
\hline Total & 1178 & & \\
\hline
\end{tabular}




\section{LES PRODUCTIONS}

\section{CARACTÉRISTIQUES TECHNIQUES}

\section{Cuisson}

Cuites vraisemblablement en fosse, les céramiques issues de l'atelier de la Sarra présentent généralement les couleurs sombres correspondant à ce type de structure. En effet $84 \%$ d'entre elles attestent une cuisson en atmosphère réductrice et $16 \%$ une cuisson en mode A (Picon, 1973).

\section{PÂtes ARgileuses}

Deux pâtes différentes ont été choisies à la Sarra pour la fabrication des céramiques culinaires. Elles comportent toutes deux des dégraissants d'origine granitique (quartz, feldspath, mica) cn assez grande quantité, mais elles diffèrent par l'abondance des moyennes et des grosses inclusions. Néanmoins les pâtes de la Sarra ont un aspect brut, comme si les potiers ne leur avaient pas fait subir de traitement entre l'extraction et le façonnage.

\section{TYPOLOGIE}

LES FORMES BASSES (pl. 14)

\section{Type 1}

Les écuelles : 244 tessons dont 37 en mode A et 127 en mode B ; 34 formes dont 3 en mode A et 31 en mode B (pl. 14, n" 4 à 7 ).

Les écuelles sont à fond plat ou légèrement soulevé et à paroi oblique ; la lèvre simple forme parfois un bourrelet externe ou présente une rainure interne. L'intérieur de l'écuclle est très souvent enduit d'un engobe micacé. Deux modules sont présents : les écuelles de petites dimensions, dont le diamètre d'ouverture varie entre 12 et $14 \mathrm{~cm} \mathrm{(n"4)}$ et les plats, dont le diamètre varie entre 23 et $33 \mathrm{~cm}\left(\mathrm{n}^{\prime \prime} 5\right.$ à 7$)$. L.es écuelles à fond plat et paroi oblique sont utilisées à Lyon (Desbat et al., 1979, pl. IV, $\mathrm{n}^{\circ *} 4$ et 5) et à Vienne (Godard, 1992a) dès l'époque augustécnne et jusqu'au début du II ${ }^{*}$ s. Les variantes observées sur les bords ne semblent pas représentatives d'une évolution chronologique.

\section{Type 2}

Les marmites à fond bombé du type Haltern 56 : 28 tessons ; 4 formes (pl. 14, n" 8). Elles offrent des dimensions très variées : le diamètre d'ouverture est de $13,5 \mathrm{~cm}$ pour la plus petite et de $30 \mathrm{~cm}$ pour la plus grande. La plus petite des marmites est recouverte sur les parois internes et sur la lèvre d'une matière blanche qui pourrait être un engobe micacé surcuit. Ce type de marmite est représentatif de la période augustéenne aussi bien à Lyon (Desbat et al., 1979, pl. IV, n"11) et à Vienne (Godard, 1992a, pl. X, n" 126) que dans les camps rhénans: Oberaden (l.oeschcke, 1942, pl. 33, n66), Haltern (Loeschcke, 1909, type 56) ou à Besançon (Guilhot et al., 1992, p. 281, n”230).

\section{Type 3}

Une marmite de forme unique cuite en mode A : 1 tesson (pl. 14, no 9). C'est une forme peu fréquente repérée dans un contexte un peu plus récent sur le site de la rue des Farges à Lyon (Desbat et al., 1979, pl. VII, n"8).

\section{Type 4}

Une marmite cuite en mode A : 2 tessons (pl. 14, $\left.\mathrm{n}^{\prime \prime} 10\right)$.

\section{Type 5}

I.es brûle-parfum (Aschenbecher) : 92 tessons ; 5 formes (pl. 15, n"1). Ainsi dénommés car il a été observé, sur les sites de consommation, que ces vases portaient des traces de brûlure sur la paroi interne, comme s'ils avaient contenu des braises. La panse carénée est souvent soulignée de gorges, le pied est haut et la lèvre fréquemment digitée. Un engobe de mica recouvre la paroi interne au niveau de la lèvre et la paroi externe jusqu'à la carène. Cette forme est toujours cuite en mode A. Leurs dimensions sont à peu près constantes, le diamètre d'ouverture est compris entre 17 et $19 \mathrm{~cm}$. Le brûle-parfum est une forme qui apparaît à Lyon dans un contexte de la rue des Farges daté des années 10-30 après J.-C. (Desbat el al., 
1979, pl. VII, n"11) ; elle est ćgalement présente à Neuss (Filtzinger, 1972, pl. 45, n“ 4 à 7) et à Colchester (Hull, 1963, type 198).

\section{Type 6}

I.es couvercles : 74 tessons dont un en mode A; 22 exemplaires dont un en mode A (pl. 15, n" 2 à 4) sont de forme simple avec bouton de préhension; le bord est plat parfois légèrement relevé. Ils ne portent aucune trace d'engobe micacé. Aucun module particulier n'a été constaté (variation du diamètre entre 7 et $27 \mathrm{~cm}$ ). Seules deux formes sont de grandes dimensions (37 ct $40 \mathrm{~cm}$ de diamètre). Elles pouvaient être utilisées autant comme plat que comme couvercle.

\section{LES FORMES HAUTES ( $\mathrm{pl} .15$ et 16)}

\section{Type 7}

Les pots ovoïdes à col côtelé : 112 tessons dont 2 en mode A ; 17 exemplaires dont 2 en mode A (pl. 15, n“ 5 et 6). Cette forme, à fond plat et large ouverture dont la lèvre retournée et le col « côtelé » sont micacés, était utilisée en cuisine pour la cuisson ou la conservation d'aliments mais également comme urne cinéraire. Le diamètre d'ouverture est très variable : de 14 à $29 \mathrm{~cm}$. Un vase dont le diamètre atteint $36 \mathrm{~cm}$ est d'ailleurs muni d'au moins une anse (pl. 15, n"6). I e pot ovoïde à col côtelé est l'une des formes en céramique commune la plus répandue en Gaule romaine de la fin du ${ }^{\mathrm{cr}} \mathrm{s}$. avant J.-C. à la fin du $I^{\mathrm{rr}}$ s. après J.-C. : à I.yon (Desbat $e t$ al., 1979, pl. IV, n* 8; Genin, 1993), à Vienne (Godard, 1992a, pl. XI, n”s 132 et 133), en Bourgogne (Joly, 1994, p. 38, n" 22 et 23), à Besançon (Guilhot et al., 1992, p. 234, n"180). Elle est fabriquée aussi à Aoste, Isère (Iaroche, 1987 : type 1 de la céramique communc sombre). Sans toutefois atteindre la taille des pots augustéens, qui d'ailleurs sont très souvent en céramique non tournée, les pots à col côtelé de cet atelier sont encore de grandes dimensions; en revanche, à la fin du $\mathrm{I}^{\mathrm{er}} \mathrm{s}$. après J.-C., cette forme est plus petite.

\section{Type 8}

Les pots cylindriques à col côtelé : 20 tessons ; 2 formes (pl. 15, n*7). Comme le type précédent ce vase présente un enduit micacé sur le col. Diamètre de l'ouverture : $14 \mathrm{~cm}$.

\section{Type 9}

Les pots à col caréné : 133 tessons ; 12 formes (pl. 16, $\left.n^{\prime \prime} 1\right)$. Ils ont un fond plat, un col couvert d'un enduit micacé et ont la même fonction culinaire que les pots ovoïdes à col côtelé, mais une durée de vie plus courte car ils disparaissent à I yon à partir du milieu du $\mathrm{I}^{\mathrm{er}} \mathbf{s}$. (Desbat et al., 1979, pl. III, n*10 ; Genin, 1993).

\section{Type 10}

Un pichet à col tronconique : 1 tesson et 1 forme. Ils sont de forme ovoïde et à fond plat présentant sur le col un décor lissé de lignes verticales. Une anse en ruban part de la lèvre légèrement déversée et se rattache sur la panse (diamètre d'ouverture : $12 \mathrm{~cm}$ ) (pl. 16, no2). C'est le pichet en céramique commune sombre le plus fréquent dans les contextes lyonnais du I" s. (Desbat et al., 1979, pl. IV, n6). Il apparaît dès l'époque augustéenne à I.yon (Genin, 1993) mais aussi à Chalon-sur-Saône (Augros, 1984, fig. 11).

\section{Type 11}

Un pichet à col tronconique : 1 tesson sans décor lissé sur le col; cuit en mode A (pl. 16, n"3).

\section{Type 12}

Un pichet non identifiable car trop fragmentaire : 6 tessons.

\section{Type 13}

Les cruches à bec tréflé : 57 tessons ; 4 formes (pl. 16, n"4). Bien que fréquemment désigné sous le nom de cruche, ce type de récipient a en réalité une fonction de bouilloire, comme le montrent les traces de feu et le dépôt de calcaire souvent observé à l'intérieur des vases usagés. Cette fonction explique pourquoi ils sont toujours fabriqués en pâte siliceuse (Batigne, Desbat, 1996). I es exemplaires de l'atelier ont une panse très carénée et un fond plat; le col tronconique se resserre sous une embouchure évasée et pincée ; l'anse en ruban part de la 
lèvre en bandeau et se rattache sur l'épaule très plate. À I.yon, cette cruche accompagne presque toujours le pichet de type 11 (Desbat et al., 1979, pl. VI, n"1 ; Genin, 1993). Elle est présente à Vienne dans la réserve de céramiques d'époque claudienne (Codard, 1992a, pl. XI, no 143).

\section{Type 14}

Deux anses en ruban micacées non attribuables : 4 tessons.

\section{Type 15}

Un pot à boutures : 1 tesson (pl. 16, n"5). C'est un pot perforé de trous avant cuisson : un sur le fond et les autres sur la paroi ; à Saint-Romain-en-Gal le péristyle 17 de la Maison des Dieux Océan a livré le long du bassin un alignement de pots identiques interprétés comme des pots à bouture (Desbat et al., 1994) ; leur présence est attestée sur plusicurs sites lyonnais (Desbat, à paraître).

\section{Type 16}

I.es creusets : 3 fonds (pl. 16, n"6). Ce sont des formes hautes fermées dont les parois sont très épaisses. I a paroi externe est inciséc de lignes verticales pour permettre une meilleure adhésion de la gangue de terre. Ils sont cuits en mode A. Des découvertes récentes effectuées à Vaise ont confirmé l'utilisation de tels récipients pour la cémentation du laiton (Picon et al., 1995).

\section{DATATION}

Cet atclier aurait fonctionné à la fin du règne de Tibère et au débu de celui de Claude. I es éléments de datation autres que la céramique commune sont raress scul un Drag. 11 en sigillée de la Graufesenque (pl. 14, n"1), provenant de la lentille dépotoir, permet de proposer une datation, confirmée d'ailleurs par la céramique commune dont les types sont courants sur les sites lyonnais d'habitat (Desbat et al., 1979).

Cette production de céramiques communes de l'atelier lyonnais de la Sarra est typique, par ses formes, des années 30-40 après J-C. (Desbat et al., 1979). Dans ce dépotoir, on peut noter l'absence des formes de céramiques communes qui apparaissent sur les sites d'habitat lyonnais vers les années 50-60 après J-.C., comme les vases carénés à décor de lignes verticales lissées sur la panse et à tenons de préhension (Desbat et al., 1979, pl. VII, n" 1 et 2), les petits pots à lèvre courte, soulignée de gorges et dont la panse est également décorée de lignes verticales lissées (1)esbat et al., 1979, pl. VI, n"7), ou la forme évoluće de la marmite à fond bombé (Desbat et al., 1979, pl. VII, n"3), cuite alors en mode A. Il est également à signaler l'absence de certaines formes typiques des contextes augustéens comme les écuelles à bord rentrant (1)esbat et al., 1979, pl. I, n” 5 et 6). Ia plupart des types de l'atelier se trouvent dans la réserve de céramiques de Vienne, datée de l'époque de Claude : les pots ovoïdes à col côtelé (Godard, 1992a, pl. XI, n"133), les couvercles (Godard, 1992a, pl. XI, n"s 130 et 131), les pots carénés (Godard, 1992a, pl. XII, n"139), les "cruches à bec tréflé " (Godard, 1992a, pl. XII, n"143). Tous ces éléments typologiques confirment bien la datation indiquée par la présence du Drag. 11 en sigillée de la Graufesenque. 

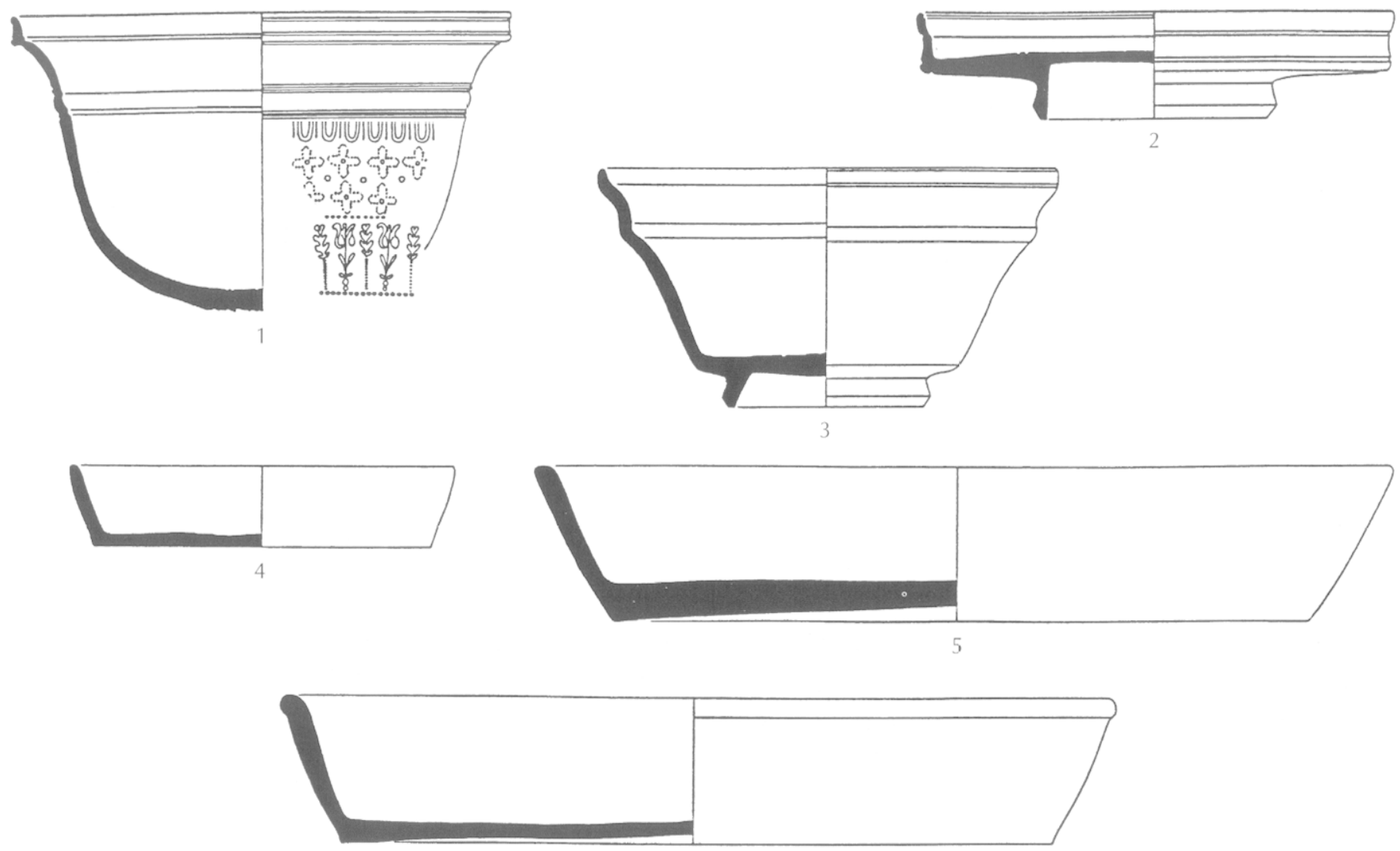

6
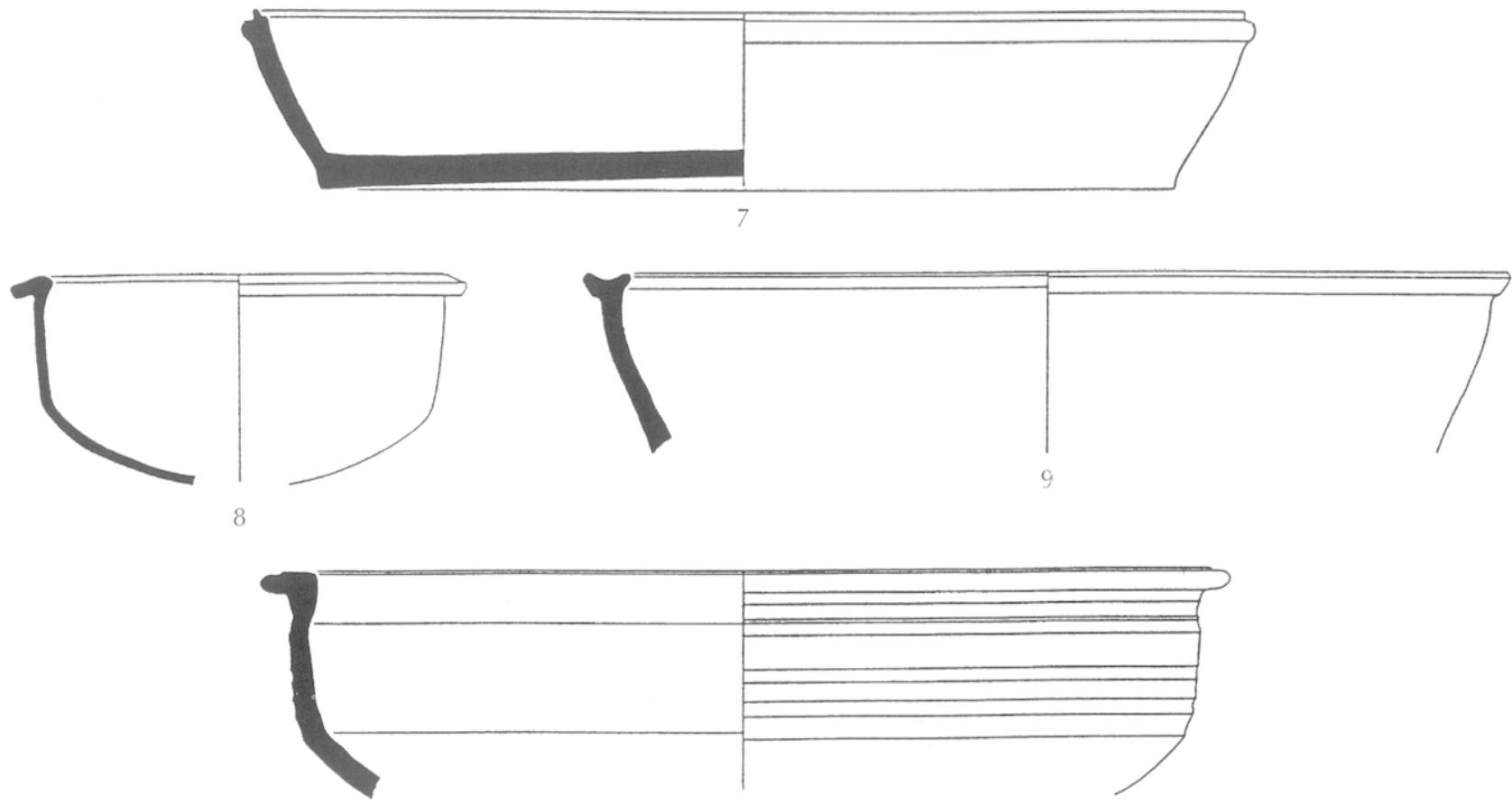

10

P1. 14. Céramiques de l'atelier de la Sarra : 1,2, sigillées; 3, imitation de sigillée; 4-10, céramiques communes sombres (échelle: 1/3). 


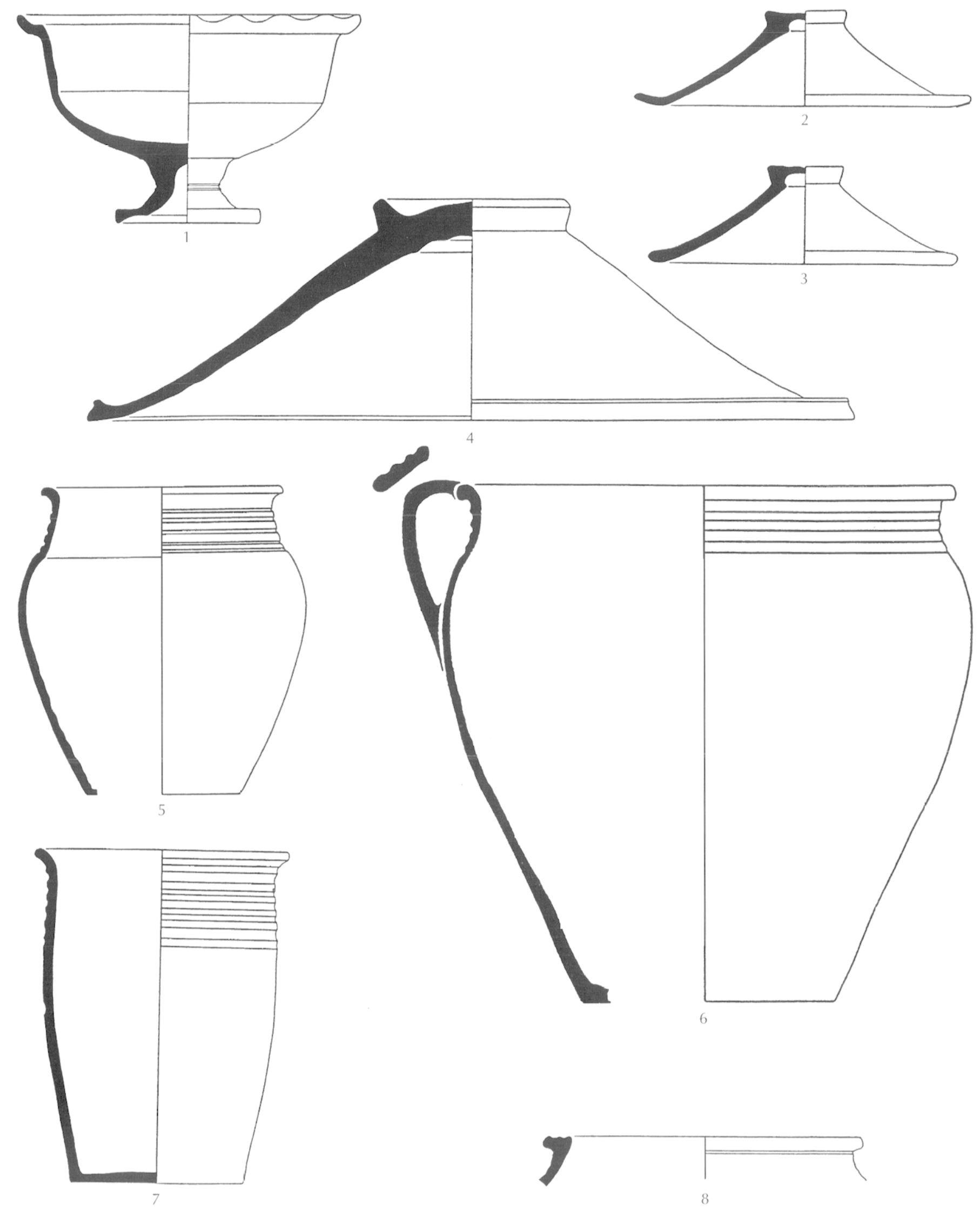

Pl. 15. Céramiques communes sombres de l'atelier de la Sarra (échelle : 1/3). 

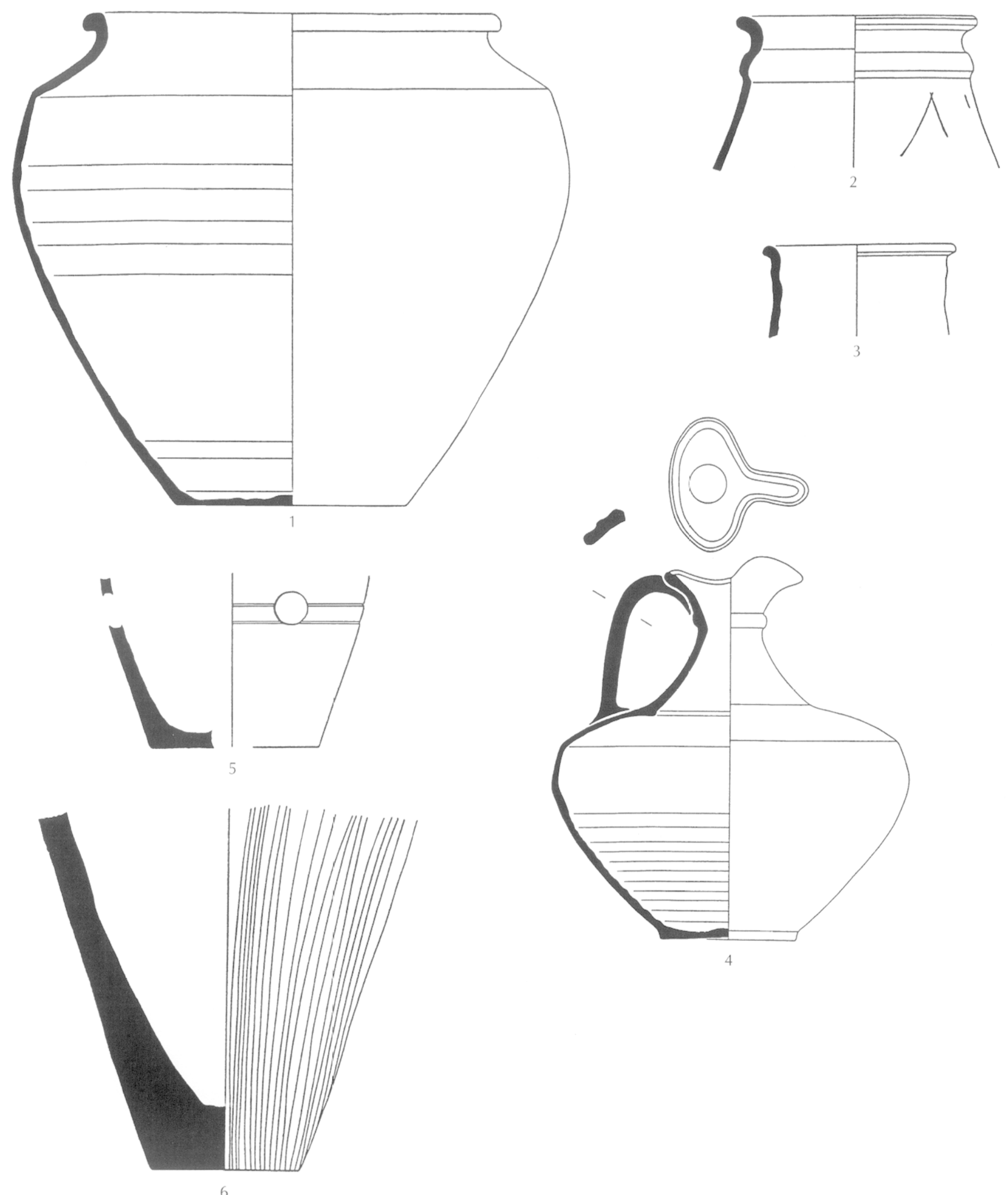

PI. 16. Céramiques communes sombres de l'atelier de la Sarra (échelle: 1/3). 